reviewed with regard to indication, effect on disease activity and acute phase response, adverse events and patient global assessment.

Results: There were 43 patients with FMF $(20 \mathrm{M} / 23 \mathrm{~F})$ who were treated with Anakinra for various indications (colchicine resistant recurrent febrile attacks in 39 , colchicine related side effects in 3 , both in 1 ). The mean age of the patients was $31.73 \pm 9.56$ years. The mean duration of the disease was $19.90 \pm 10.52$ years. There were various co-existing pathologies among this study group like Ankylosing Spondylitis (4), Psoriasis (1), Behçet's disease (1), Gout (1), Vasculitis (1), Adult-onset Still's disease (1), Polyarthritis Nodosa (1) and Celiac disease (1). The mean colchicine dose was $1.84 \pm 0.31 \mathrm{mg} / \mathrm{d}$. As for the dosage, 35 patients were on $100 \mathrm{mg} /$ day, 6 were $100 \mathrm{mg}$ on alternate days, 2 were on $200 \mathrm{mg} /$ day. The mean duration of anakinra treatment was $10.76 \pm 13.64$ months. After the initiation of anakinra 29 patients became attack-free, 9 patients reported more than $50 \%$ decrease, 3 patients less than $50 \%$ decrease, and 2 patients no change in the frequency of the attacks. Mean patient global assessment decreased from $7.55 \pm 2.34$ to $2.82 \pm 2.63$ under Anakinra treatment $(p<0.001)$.

As for the adverse events, eight patients (\%18) had allergic reactions under Anakinra treatment (severe disseminated rash in 1 patient and severe injection site reaction in 4 patients and tolerable injection side reaction in 3 ) which necessitated termination of treatment in 5 patients. Anakinra was stopped because of genital warts and urinary tract infection in one other patient. Worsening of psoriatic lesions was observed in another patient. There were no adverse events in the remaining 41 patients during the course of treatment. On the other hand, treatment was terminated due to inadequate response in 11 $(25 \%)$, remission in 2 and patient preference in 3 patients. Nineteen patients are still on Anakinra treatment for $10.09 \pm 14.51$ months.

Conclusions: Anakinra is an effective and relatively safe alternative treatment in FMF patients with inadequate response or intolerance to colchicine, however approximately one fourth of the patients stop anakinra for insufficient response.

Disclosure of Interest: None declared

DOI: 10.1136/annrheumdis-2017-eular.5436

\section{FRI0597 FATIGUE IN FAMILIAL MEDITERRANEAN FEVER (FMF) AND ITS RELATIONS WITH OTHER CLINICAL PARAMETERS}

\section{Unal Ertekin ${ }^{1}$, T. Duruoz ${ }^{1}$, D. Karali ${ }^{2}$, F. Ulutatar ${ }^{2} .{ }^{1} P M \& R$ Department,} Rheumatology Division; ${ }^{2} P M \& R$ Department, Marmara University, School of Medicine, Istanbul, Turkey

Background: Fatigue is a common problem in patients with rheumatic disease. It may cause disability and poor quality of life (1). Although fatigue and its determinants are studied in several rheumatic diseases, there is no study in Familial Mediterranean Fever (FMF).

Objectives: The aim of this study is to investigate fatigue in FMF patients as a disabiling symptom and its associations with clinical and demographic variables. Methods: FMF patients were recruited into the study according to FMF Tel Hashomer criteria (2). Control group composed of healthy individuals. Patients who were pregnant or who had concomitant medical illnesses such as cancer, fibromyalgia, or psychiatric conditions such as psychosis or bipolar disorder were excluded. Age, gender, disease duration, education, marital status were noted as demographic features. Number of attacks in the last year, type of attack, involvement of joints, dosage of colchicine, genotype, amyloidosis, and severity of FMF was assessed with PRAS score, visual analogue score of pain (VAS-pain) and VAS-fatigue were used as clinical parameters. Pittsburgh Sleep Quality Index (PSQI), Multidimensional Assessment of Fatigue (MAF), Nottingham Health Profile (NHP), Fatigue Severity Scale (FSS), Fatigue Impact Scale (FIS) and Hospital Anxiety and Depression Scale (HADS) were filled out by both control and study group. Assessment of normality was analyzed with Shapiro-Wilk test. Differences in the mean scores of control and study group were compared with independent samples Mann-Whitney $U$ and Kruskal-Wallis test. Relationship between continuous variables was assessed with Spearman's correlation coefficient (rho).

Results: 61 FMF patients and 61 age, gender (44 female, 17 male in each group) matched controls were enrolled into the study. Mean age of FMF and control group were $35.5 \pm 11.8$ and $35.8 \pm 11.7$ years, respectively. The mean disease duration was $82.5 \pm 81.7$ months. Difference between mean of VAS-pain, VAS-fatigue, PSQI total score, MAF, all subsets of NHP, FSS, FIS, HADS scores of FMF patients were significantly higher than control group $(p<0.0001)$. The correlations between scales assessing fatigue and other outcome measures in FMF patients was shown in Table.

\begin{tabular}{lcccc}
\hline Spearman'sCorrelation (rho) & VAS.fatigue & MAF & FSS & FIS \\
\hline PSQI.TotalScore & $0.49^{\star \star \star}$ & $0.53^{\star \star \star}$ & $0.38^{\star \star}$ & $0.50^{\star \star \star}$ \\
NHP.EnergyLevel & $0.43^{\star \star \star}$ & $0.65^{\star \star \star}$ & $0.54^{\star \star \star}$ & $0.55^{\star \star \star}$ \\
NHP.Pain & $0.58^{\star \star \star}$ & $0.66^{\star \star \star}$ & $0.56^{\star \star \star}$ & $0.67^{\star \star \star}$ \\
NHP.EmotionalReaction & $0.40^{\star \star}$ & $0.54^{\star \star \star}$ & $0.48^{\star \star \star}$ & $0.65^{\star \star \star}$ \\
NHP.Sleep & $0.32^{\star}$ & 0.24 & 0.12 & $0.31^{\star}$ \\
NHP.Sociallsolation & $0.27^{\star}$ & $0.39^{\star \star}$ & $0.50^{\star \star \star}$ & $0.54^{\star \star \star}$ \\
NHP.PhysicalAbilities & $0.51^{\star \star \star}$ & $0.64^{\star \star \star}$ & $0.56^{\star \star \star}$ & $0.69^{\star \star \star}$ \\
HADS.Anxiety & $0.43^{\star \star \star}$ & $0.55^{\star \star \star}$ & $0.52^{\star \star \star}$ & $0.75^{\star \star \star}$ \\
HADS.Depression & $0.30^{\star}$ & $0.42^{\star \star \star}$ & $0.42^{\star \star \star}$ & $0.57^{\star \star \star}$ \\
\hline$\star \star \star * *$
\end{tabular}

${ }^{* \star *}=p<0.001 ;{ }^{* \star}=p<0.01 ;{ }^{*}=p<0.05$.

Conclusions: This study has shown that fatigue in FMF is associated with a number of psychological, sleep, quality of life and disease related factors. FMF group had increased pain, fatigue, sleep disturbance and decreased quality of life compared to control group. FMF patients with fatigue may benefit from pharmacological and psychological interventions which target these factors.

References:

[1] Nikolaus, Stephanie, et al. Fatigue and factors related to fatigue in rheumatoid arthritis: a systematic review. Arthritis care \& research 65.7 (2013): 1128-1146.

[2] Livneh, Avi, et al. Criteria for the diagnosis of familial Mediterranean fever. Arthritis \& Rheumatology 40.10 (1997): 1879-1885.

Disclosure of Interest: None declared

DOI: 10.1136/annrheumdis-2017-eular.4792

\section{FRI0598 CHARACTERIZATION OF PATIENTS WITH AN INITIAL DIAGNOSIS OF UNDIFFERENTIATED CONNECTIVE TISSUE DISEASE. OBSERVATIONS FROM A LONGSTANDING MONOCENTRIC COHORT}

C. Sepúlveda ${ }^{1,2,3}$, A.V. Taulaigo ${ }^{2,3}$, F. Carreiro ${ }^{2,3,4}$, M.F. Moraes-Fontes ${ }^{2,3}$. ${ }^{1}$ Serviço de Medicina, Centro hospitalar Médio Tejo, Abrantes; ${ }^{2}$ Unidade de Doenças Auto-imunes/Medicina 7.2, Hospital de Curry Cabral, Centro Hospitalar de Lisboa Central; ${ }^{3}$ NEDAl - Núcleo de Estudos de Doenças Autoimunes da Sociedade Portuguesa de Medicina Interna, Lisbon; ${ }^{4}$ Serviço de Medicina, Hospital do Divino Espírito Santo, Ponta Delgada, Azores, Portugal

Background: Evolution from Undifferentiated Connective Tissue Disease (UCTD) to a defined Autoimmune Disease (AID) remains unexplained and usually occurs in the first 3 to 5 years.

Objectives: To determine the frequency and predictors of differentiation from UCTD to AID in a selected cohort of patients originally labelled as UCTD within the first 3 years of follow-up.

Methods: Demographic and clinical features were retrieved: (i) retrospectively, from the AID's Unit current database in December $2016(n=195)$, including Systemic Lupus Erythematosus (SLE), incomplete SLE, Sjögren's Syndrome (SS), Systemic Sclerosis (SSc), VEDOSS and Mixed Connective Tissue Disease (MCTD); and (ii) from a prospectively UCTD database created in January 2012 $(n=48)$. Inclusion criteria for the latter pertains to ANA positive patients, not fulfilling any of the existing classification criteria for AID and without severe organ involvement [1]. Patients with cutaneous lesions suggestive of lupus, inflammatory myopathies, erosive arthritis, systemic sclerosis and certain auto-antibody profiles were excluded a priori from the definition of UCTD as these are thought to herald defined conditions, as previously defined [2]. Definition of stable UCTD includes patients with at least one clinical manifestation of AID, positive ANA result and disease duration of at least 3 years [3]. Comparisons between stable UCTD and progressing patients were made using Wilcoxon Rank Sum and Chi square tests, $p$ values of $<0.05$ were considered statistically significant.

Results: Each individual patient was analysed for differentiation into AID at yearly intervals. Overall, the main features of the prospective UCTD cohort were arthralgia $(79 \%)$, rash $(31 \%)$, arthritis $(19 \%)$, sicca symptoms $(19 \%)$, photosensitivity $(17 \%)$ and Raynaud $(13 \%)$. Prospective analysis in the UCTD cohort revealed differentiation in $4 / 48$ patients (8.3\%): into rheumatoid arthritis $(n=2)$, psoriatic arthritis $(n=1)$ and SLE $(n=1)$. The main difference between stable UCTD and those that progressed to AID was the presence of arthritis $(p=0.003)$. Median age of onset and symptom duration was similar between both groups. Retrospective analysis yielded very few patients presenting as UCTD ( $n=5 / 195)$ : 2/106 SLE; $1 / 13$ incomplete SLE; $1 / 43$ SS; $1 / 19$ SSc; 0/7 VEDOSS and 0/7 MCTD with no distinguishing features.

Conclusions: Very few patients differentiated in the UCTD cohort after 3 years of follow-up and in our retrospectively studied cohort, in accordance to a previous study [4]. Apart from arthritis, there were no other predicting factors for differentiation to AID. UCTD at disease onset seems to be a rare event.

References:

[1] Mosca M, et al. Clin Exp Rheumatol. 1999;17(5):615-20.

[2] Mosca M, et al. Lupus. 2008;17(4):278-80.

[3] Mosca M, at al. J Autoimmun. 2014:48-49:50-2

[4] Danieli MG, et al. Clin Exp Rheumatol. 1999;17(5):585-91.

Disclosure of Interest: None declared

DOI: 10.1136/annrheumdis-2017-eular.5495

\section{FRI0599 A RETROSPECTIVE OVERVIEW OF CLINICAL FEATURES, SEROLOGY AND HISTOLOGY OF IGG4 RELATED DISEASE IN HONG KONG: A DATASET OF 108 PATIENTS FROM FOUR REGIONAL HOSPITALS}

C.P. Tang ${ }^{1}$, K.L. Lee ${ }^{1}$, K.W. Lee ${ }^{2}$, W.L. $\mathrm{Ng}^{3}$, M.C. Wan ${ }^{4}$, K.Y. Yuen ${ }^{5} .{ }^{1}{ }^{M e d i c i n e}$, PYNEH; ${ }^{2}$ Medicine, HKSH; ${ }^{3}$ Medicine, UCH; ${ }^{4}$ Medicine, $R H ;{ }^{5}$ Medicine, QMH, HK, Hong Kong

Background: IgG4 related disease (IgG4RD) is a spectrum of immune mediated disease with multiple organs involvement characterized by organ enlargement, function loss or obstructive symptoms $(1,2)$

Objectives: In this retrospective study, clinical, serological and histological features of IgG4RD patients over the past ten years from four regional hopsitals were reviewed. 